\title{
Research Paper: Effect of Mirror Therapy on Upper Limb Function: A Single Subject Study
}

\author{
Seyed Alireza Derakhshanrad ${ }^{1,2 *}$, Emily Piven ${ }^{3}$, Bahareh Zeynalzadeh Ghoochani ${ }^{1}$, Safoora Toosi $^{1}$ \\ 1. Department of Occupational Therapy, School of Rehabilitation Sciences, Shiraz University of Medical Sciences, Shiraz, Iran. \\ 2. Rehabilitation Sciences Research Center, Shiraz University of Medical Sciences, Shiraz, Iran. \\ 3. Department of Rehabilitation Sciences, College of Health Sciences, University of Texas at El Paso, Texas, United States.
}

\begin{tabular}{|c|c|}
\hline $\begin{array}{l}\text { Use your device to scan } \\
\text { and read the article online }\end{array}$ & \\
\hline 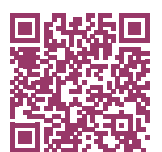 & $\begin{array}{l}\text { Cltation: Derakhshanrad SA, Piven E, Zeynalzadeh Ghoochani B, Toosi S. Effect of Mirror Therapy on Upper Limb Function: } \\
\text { A Single Subject Study. Iranian Rehabilitation Journal. 2017; 15(3):227-234. https://doi.org/10.29252/NRIP.IRJ.15.3.227 } \\
\text { dof: https://doi.org/10.29252/NRIP.IRJ.15.3.227 }\end{array}$ \\
\hline
\end{tabular}

Article info:

Received: 10 Mar. 2017

Accepted: 29 Jun. 2017

Keywords:

Cerebral palsy, Hemiplegia,

Mirror therapy, Neuroscience

\section{A B S T RACT}

Objectives: Mirror therapy is a unique treatment with a touch of modality that is purported to improve the motor function of the affected limb in individuals with hemiplegia. Previous studies have focused on the neuro-physiological factors underlying the mechanism of the clinical effect of this technique. The present study aims to understand the mechanism using the rehabilitation method and neuro-occupation model as well as analyze the effects of mirror therapy on the upper limb function of subjects with spastic hemiplegic cerebral palsy.

Methods: Single subject design known as withdrawal design was used by a convenience sample of four subjects. The study involved three observational phases known as baseline, treatment, and withdrawal phases that took place during a 10 week period. The study contained a home-based mirror therapy protocol whereby the participants were instructed to do some exercises on a daily basis. The improvement of the hand function of the hemiplegic side was examined by Box and Block test along with two more activities including Threading Beads and Stacking Rings.

Results: The ability to perform the Box and Block test, Threading Beads, and Stacking Rings tended to remain steady in the baseline phase, whereas there was a noticeable improvement during the treatment phase and a decline in the withdrawal phase.

Discussion: From the perspective of visual feedback neuro-occupation model, it could be hypothesized that alterations to the sensory system caused by the mirror reflection of non affected hand may have led to the destabilization of the sensory cortices that changed the participants' intention, meaning, and perception, thereby improving the subject's motor control.

\section{Introduction}

W

hen conceptual frameworks or models merge with practice and identify evidences of measured outcomes resulted from therapies, the clients are the ultimate beneficiaries [1]. Therapeutic Models help the rehabilitation therapists (i.e. occupational therapists) in rationalizing the observed therapeutic phenomena in order to comprehend the mechanism and speculations behind such therapeutic

* Corresponding Author:

Seyed Alireza Derakhshanrad, PhD

Address: Department of Occupational Therapy, School of Rehabilitation Sciences, Shiraz University of Medical Sciences, Shiraz, Iran.

Tel: +98 (917) 7181002

E-mail:derakhshan@sums.ac.ir 
interventions [1]. Mirror Visual Feedback (MVF), also known as mirror therapy, is one such therapeutic intervention that lacks research to identify the underlying mechanism and speculations behind its beneficial clinical effects [2]. Thus, it would be of interest to make the mechanism underlying the mirror therapy and its clinical effects more transparent through a conceptual framework.

Mirror therapy is a unique treatment with a touch of modality that is purported to improve the motor function of the affected limb in adults with hemiplegia following a cerebrovascular accident (stroke) [3] and children with spastic hemiparetic cerebral palsy $[4,5]$. The technique uses a mirror that is placed between two limbs, thus enabling a client to view the mirror image of movements of the non-affected limb superimposed on the contralateral affected limb [3]. Although no model was utilized to interpret the phenomenon of improving motor control, research on children supports the hypothesis that the brain might benefit from the feedback received from the mirror therapy resulted by the increased excitability of the primary motor cortex of the brain's affected hemisphere $[4,5]$.

The theoretically-driven model used in this research to interpret the mechanism underlying clinical effects of mirror therapy is called the Neuro-occupation Model. This is one of the most recent models in occupational therapy which improves one's ability to function following a disease or injury that interrupts the functionality of an individual's daily living skills. This model accepts the key underpinning concept that our occupation shapes development of the brain, which changes its function and vice versa [6]. It also describes a dynamic process in which occupations establish a network at three heterarchical levels in the brain, i.e. intention, meaning, and perception. They enable a human being to set goals at Intention level, make meaning at Meaning level, and raise belief or self-awareness at the Perception level [7].

Two key properties of the model, known as "perturbance" and "attractor", were substantiated by the limited qualitative research with an objective to begin the process that led the subjects with cerebro-vascular strokes to initiate a positive change in their motor control [8]. Accordingly, perturbance has been acknowledged to have contributed to positive changes by providing influential opportunities or experiences to the clients, thereby receiving appreciation as an important property for their change [8]. Attractor was reported to be a preferred mental state that enticed or attracted clients to make an intentional action, engage in meaningful occupational performance, and develop realistic beliefs that altered their perception [8].
The Neuro-occupation model was used in this study to understand how upper limb motor function of children with spastic hemiplegic cerebral palsy was influenced by the mirror therapy. This study also explains how a mirror used in MVF therapy provides the visual, kinesthetic, and proprioceptive perturbance in sensory feedback, which exerts an influence on Intention, Meaning, and Perception levels and enables the child to be attracted to refined goals, meanings, and beliefs about themselves. This refinement could ultimately trigger the changes in cognition through a continuous circular-causality cognitive process among Intention, Meaning, and Perception levels [9]. Further, the client is assumed to be in charge of his or her circular-causality cognitive process [10] and the occupational therapist provides the "just-right challenge" (perturbance) [11] to starts the process and opportunity for the client to be actively attracted to realistic intention, meaning, and perception levels [12].

Despite acknowledging the importance of sensory feedback in shaping occupational performance and vice versa [13], there are few studies that limited to the processes involved in occupational therapy [14]. To understand the linkage between sensory feedback and occupation, the present study aims to utilize the Neurooccupation model to interpret the outcomes of the mirror therapy. Therefore, a mirror will be considered as a source of sensory feedback, upon which the brain has the potential to be triggered. It might improve the motor control and occupational performance of the selected children with spastic hemiplegic cerebral palsy. The question that has been researched in this study is 'Can the Neuro-occupation model explain the relationship between mirror visual feedback (independent variable) and upper limb motor control (dependent variable)?'

\section{Methods}

Study design

A single-subject design known as withdrawal design was used for this study [15] to have a great clinical applicability in rehabilitation research where alterations in the behavior of a single individual are the target outcome that requires investigation [15]. Single-subject researchers have argued that single-subject designs have the superiority over large-group designs, such as randomized controlled trials considering that the research contentious issue of "science-versus-practice" gap is addressed in the singlesubject design compared to large-group design [16].

Withdrawal design is sometimes referred to as reversal or A-B-A design, which involves implementation fol- 

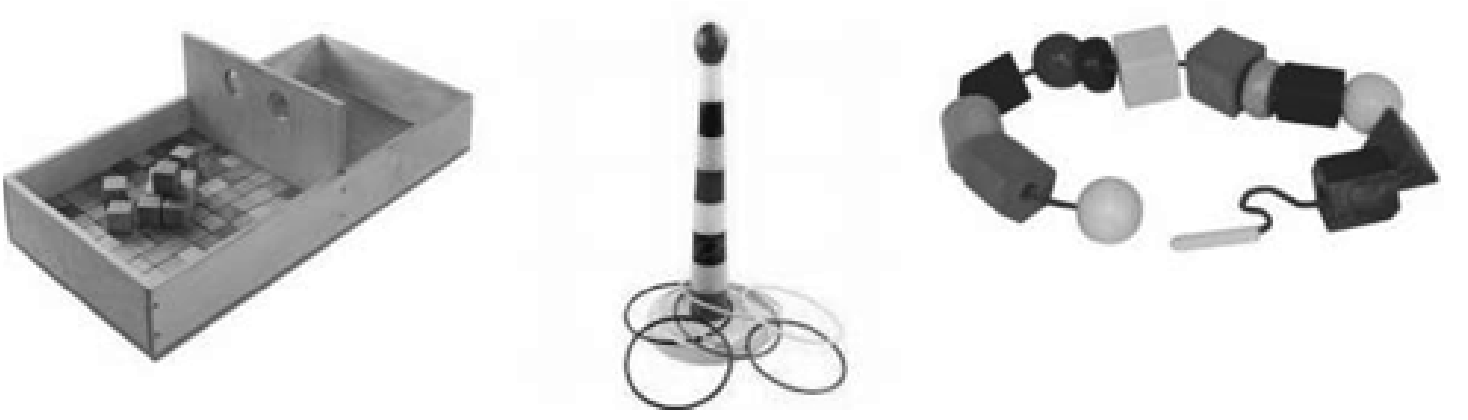

IIranlan Rkhabulltgation Journa

Figure 1 Assessment tools including the box and block test, stacking rings, and threading beads (left to right)

lowed by subsequent removal of an independent variable over the course of the study. In this case, multiple observations of the target behavior of a single individual are conducted from non-treatment phase (A) to treatment phase (B) and back to a non-treatment phase (A) $[16,17]$. The clinical trial protocol was registered in the Iranian Registry of Clinical Trial with IRCT registration number: IRCT2012080410491N1.

\section{Participants}

Four subjects ( 3 males and 1 female) were recruited from three main outpatient child rehabilitation facilities in Shiraz, Iran. Inclusion criteria were: A. Diagnosed with spastic hemiplegic cerebral palsy; B. Aged between 5 to 15 years old; C. Were cognitively-oriented (according to medical history); D. Were not having incurable vision or hearing problems (according to medical history); E. Were not treated with botulinum toxin during the last 6 months prior to the study (according to medical history); and F. Willingness of the participants and their parents to participate in the study. Exclusion criteria were: A. Unwillingness of the participants or their parents to begin or continue the trial; and B. Any reason leading the participant to be unable to continue the trial such as getting admitted to hospital or illness.

\section{Outcome measures}

The improvement of motor function of the affected hand was the main outcome (dependent variable) that was examined by a functional test called the Box and Block test. In order to make the evaluations more childfriendly, two toy activities were also used that included Threading Beads and Stacking Rings (Figure 1). All participants sat behind a table while being tested, and the three aforementioned tests allowed the examiner to evaluate the functional status of the affected upper limb and its capability in doing activities. Participants were given 1 minute for each of the three tests to be performed. The scores were based on the number of blocks transferred between two boxes, the number of rings stacked, and the number of beads threaded. The higher scores in each test were attributed to the better functional status.

\section{Intervention}

This study involved three consecutive phases, namely baseline ( 2 weeks), treatment ( 6 weeks), and withdrawal ( 2 weeks), which lasted for 10 weeks. The intervention contained a home-based mirror therapy protocol in which the participants were instructed to do a 20-minute daily regimen using a mirror $(38 \times 28 \mathrm{~cm})$ placed in front of them at an angle of $70^{\circ}$ to $80^{\circ}$ to the trunk level. The intervention protocol comprised of three parts including some exercises and activities. Exercises included practicing the range of motion and strengthening of fingers and forearms, whereas the activities included some fine motor tasks. While looking at the mirror reflection of their non-affected hand, the participants were instructed to perform the intervention protocol with both hands and arms simultaneously, though the affected hand was neither moved nor seen easily. The intervention protocol included: 1. Forearm pronation and supination; 2. Wrist and finger flexion and extension; 3. Index-thumb opposition; 4. Squeezing a foam; 5. Using a water spray; 6 . Pinching coin; and 7. Wiping a table with a piece of cloth.

\section{Procedure}

This trial was ethically approved by ethical committee of Shiraz University of Medical Sciences (ethical code: IR.SUMS.REC.1394.73). Prior to the study, the participants and their family were informed about the research and they gave consent for their children to participate in the study. All outcome measures were evaluated 7 times during the trial. To be sure about the established motor function behavior, two points of data were collected during a 2-week period in the baseline phase (A) prior to presenting the independent variable (mirror) (week 0 , $\mathrm{T}_{1}$; week $2, \mathrm{~T}_{2}$ ). The presentation of independent variable took place in the treatment phase $(\mathrm{B})$ when three points 


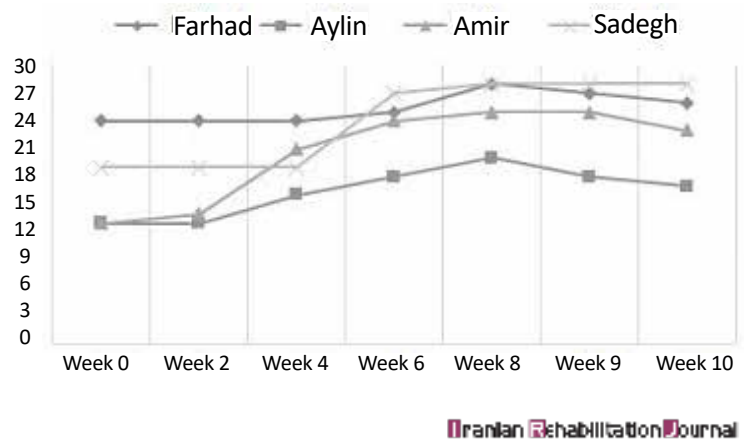

Figure 2. Changes in the participants ability to perform the box and block test

of data were collected during a 6 -week period (week 4 , $\mathrm{T}_{3}$; week $6, \mathrm{~T}_{4}$; week $8, \mathrm{~T}_{5}$ ). After completing the treatment phase, the trial continued for an extra 2 weeks without the independent variable. Two more evaluations were performed during the withdrawal phase (week 9 , $\mathrm{T}_{6}$; week $10, \mathrm{~T}_{7}$ ).

\section{Results}

Table 1 provides the descriptive information of the study participants using pseudonyms. The Manual Ability Classification System (MACS) was used to identify the levels of interaction with objects and quality of movements [18]. Level II indicated "Handles most objects, but with somewhat reduced quality and/or speed of achievement". Level III specified "Handles objects with difficulty; needs help to prepare and/or modify activities" [18]. Spasticity was assessed by Ashworth scale. The participants grade of spasticity at the baseline phase were Grade 1: "Slight increase in muscle tone, manifested by a catch and release or by minimal resistance at the end of the range of motion when the affected part(s) is moved in flexion or extension"; Grade $1^{+}$: "Slight increase in muscle tone, manifested by a catch, followed by minimal resistance throughout the remainder (less than half) of the ROM"; Grade 2: "More marked increase in muscle tone through most of the ROM, but affected part(s)

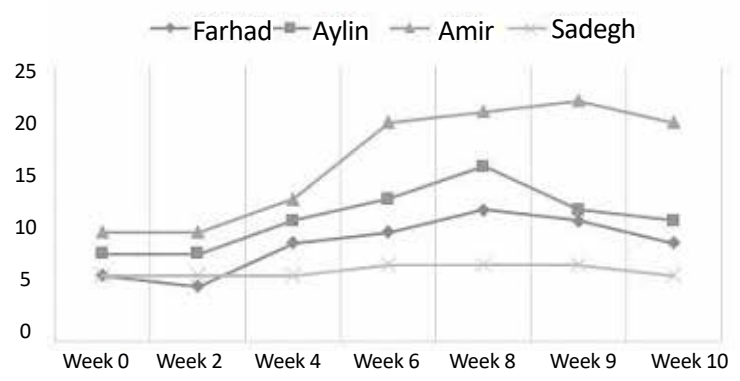

Iranlan Rehabollutron Journa

Figure 4. Changes in the participants ability to stack rings

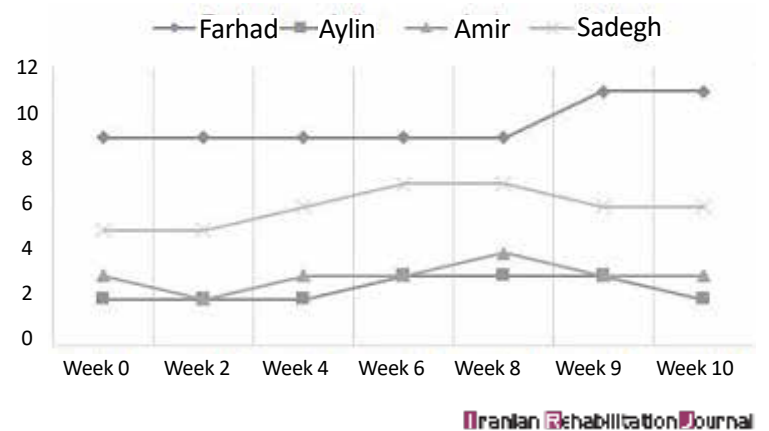

Figure 3. Changes in the participants ability to thread beads

easily moved"; and Grade 3: "Considerable increase in muscle tone, passive movement difficult" [19].

Both MACS levels and degree of spasticity (Ashworth scale) remained constant throughout the trial. However, the ability to perform the Box and Block test, Threading Beads, and Stacking Rings tended to remain steady in the baseline phase, whereas it showed a significant improvement during the treatment phase and declined in the withdrawal phase. Figures 2, 3 and 4 present the changes in participants' ability during the trial.

\section{Discussion}

The aim of this study was to analyze the clinical effects of MVF on the upper limb motor control. Hence, the change process was interpreted that occurred from a Neuro-occupation model viewpoint. Similar to the previous work, one can see the gains of the participants in this study (Figures 2, 3 and 4). A previous systematic review conducted on children and adolescents with spastic hemiplegic cerebral palsy declared that the MVF shows a promising therapeutic effect on hand function [20, 21]. In addition, Grunt et al. [22] found that subjects with spastic hemiplegic cerebral palsy gained benefit from MVF by evaluating neurophysiological evidence that showed a significant increase in the excitability of primary motor cortex, following the application of MVF in children and adolescents with hemiparesis. Grunt and colleagues evaluated the motor cortex excitability of the hemisphere, which projected to the affected hand using single-pulse transcranial magnetic stimulation (TMS). However, there has been scarce discussion on the mechanism underlying clinical effects of mirror therapy based on the theoretically-driven conceptual framework.

Clearly, there was a progression of improvement in unilateral hand skills of the instruments, which predictably declined after the short duration of treatment program (6 weeks) with cessation of MVF therapy, as it was probably not long enough to establish the habit strength. This find- 
Table 1. Descriptive information for the study participants

\begin{tabular}{cccccc}
\hline $\begin{array}{c}\text { Participants } \\
\text { (Pseudonym) }\end{array}$ & Age & Gender & Affected Side & $\begin{array}{c}\text { MACS Level } \\
\text { (Week 0) }\end{array}$ & $\begin{array}{c}\text { Modified Ashworth Scale } \\
\text { (Week 0) }\end{array}$ \\
\hline Farhad & 14 & M & Right & III & 3 \\
Aylin & 6 & F & Left & II & $1^{+}$ \\
Amir & 8 & M & Right & II & 1 \\
Sadegh & 15 & M & Right & III & 2 \\
\hline
\end{tabular}

ing is in agreement with that of Smorenburg et al. [23], and clearly showed that the positive effect of MVF was not present after the 1-week retention test, which was performed without any mirror visual feedback information. They suggested that a long-term routinely-established practice of MVF should be utilized to verify whether a long-term change takes place in movement control.

The findings of the present study were interpreted using the Neuro-occupational viewpoint for understanding the clinical effects of MVF. Lazzarini hypothesized that perturbance to the sensory system may have an effect on the destabilization of sensory cortices, which may change the participants' intention, meaning, and perception levels resulting in the improvement in their motor control [7]. Additionally, another study concluded that the sensory system of an individual plays an important role in his/ her occupation by facilitating the connection between the individual's neuron signals and whole brain activity [14].

Hence, sensory disturbances of mirror reflection therapy might be appreciated by the participants as a meaningful opportunity, which had the effect of bringing them to the state of readiness as the children and adolescents were found to respond positively to the destabilization of their sensory cortices. Each subject experienced an illusion that led to the success of the individual to initiate a goal such as, "I can visualize myself moving in this way and I can feel it happening" (as quoted by the first participant of this study). It resulted in a change in selfperception because of the experience gained in terms of motor control using the unaffected extremity. It may have first begun as an associated reaction of the affected extremity that was triggered by the sensory experience of the non-affected hand, but was not evaluated in this study.

The success may have modified the circular-causality feedback process where a new positive goal was created that fueled meaningful experiences and positive self-perception. The positive self-perception was perpetuated by the continuous sensory disturbances, which later pow- ered the continuance of intention and meaning through repetitive practice. When the treatment phase ended and practice with the mirror was curtailed, there was a decline in function after the completion of two weeks, but the four subjects did not return to baseline levels. From the Neuro-occupational perspective, a possible explanation for this finding may be that the discontinuation of mirror therapy curtailed the further development of the sensory cortices and the cessation of this treatment phase created a new perturbance.

The new perturbance included the abrupt withdrawal of the sensory, kinesthetic, and proprioceptive feedback associated with the mirror reflection, and the formation of cognitive patterns and emotional responses that provides feedback for perception. As the cognition of circular-causality process is presumed to occur continuously, whether the developing repetitive patterns in the subjects ceased or not, an emotional response was not captured by the qualitative research in the current study.

The most plausible explanation for reduction in hand function could be attributed to the short-term use of mirror therapy, as it could not overcome the behavioral pattern of the learned non-use of the affected extremity, which would take a much longer period of time. Other scholars have also suggested that MVF should be considered as an adjunct to the conventional rehabilitation of children with hemiparesis, possibly because of the duration of time it takes to alter motor behavior [23, 24].

There are many limitations of this study, namely (1) small number of participants, (2) failure to collect any qualitative data from children and their parents, (3) use of few motor assessments to gather data, and (4) lack of funding to do a neuroimaging study. Therefore, it would be of interest to do the same research with a larger sample size, and improve the validity of the study by doing triangulation in data gathering. Thus, the future research could focus on mixed method research design to gather qualitative and quantitative data simultaneously. 


\section{Conclusion}

As far as the Neuro-occupation model is concerned, the contribution of the present study is that it leads to the conclusion that alterations to the visual sensory system caused by the mirror reflection of non affected hand may have led to destabilization of the sensory cortices which changed the participants' intention, meaning, and perception, thus improving the motor control.

\section{Acknowledgments}

This research was financially supported by Shiraz University of Medical Sciences (SUMS), Iran, represents the findings of the study from an approved research project with the grant number of 93-01-06-8984. The authors would like to thank the financial support of SUMS, and the cooperation of participants and their family.

\section{Conflict of Interest}

The authors declare no conflicts of interest.

\section{References}

[1] Forsyth K, Mann LS, Kielhofner G. Scholarship of practice: Making occupation-focused, theory-driven, evidence-based practice a reality. British Journal of Occupational Therapy. 2005; 68(6):260-8. doi: 10.1177/030802260506800604

[2] Deconinck FJ, Smorenburg AR, Benham A, Ledebt A, Feltham MG, Savelsbergh GJ. Reflections on mirror therapy: A systematic review of the effect of mirror visual feedback on the brain. Neurorehabil Neural Repair. 2015; 29(4):349-61. doi: $10.1177 / 1545968314546134$

[3] Thieme H, Mehrholz J, Pohl M, Behrens J, Dohle C. Mirror therapy for improving motor function after stroke. Cochrane Database of Systematic Reviews. 2012(3):CD008449. doi: 10.1002/14651858.cd008449.pub2

[4] Gordon AL, Di Maggio A. Rehabilitation for children after acquired brain injury: Current and emerging approaches. Pediatric Neurology. 2012; 46(6):339-44. doi: 10.1016/j.pediatrneurol.2012.02.029

[5] Feltham MG, Ledebt A, Deconinck FJ, Savelsbergh GJ Mirror visual feedback induces lower neuromuscular activity in children with spastic hemiparetic cerebral palsy. Research in Developmental Disabilities. 2010; 31(6):1525-35. doi: 10.1016/j.ridd.2010.06.004

[6] Royeen CB. Chaotic occupational therapy: Collective wisdom for a complex profession. American Journal of Occupational Therapy. 2003; 57(6):609-24. doi: 10.5014/ajot.57.6.609

[7] Lazzarini I. Neuro-occupation: The nonlinear dynamics of intention, meaning and perception. British Jour- nal of Occupational Therapy. 2004; 67(8):342-52. doi $10.1177 / 030802260406700803$

[8] Derakhshanrad SA, Piven E, Zeynalzadeh Ghoochani B. Adaption to stroke: A nonlinear thinking approach in occupational therapy. Occupational Therapy in Health Care. 2017; 31(3):255-69. doi: 10.1080/07380577.2017.1335922

[9] Lazzarini I. A nonlinear approach to cognition: a web of ability and disability. In: Katz N, Baum MC, editors. Cognition and Occupation Across the Life Span: Models for Intervention in Occupational Therapy. Bethesda: American Occupational Therapy Association; 2004.

[10] Derakhshanrad SA, Piven E, Zeynalzadeh Ghoochani B Comparing the cognitive process of circular causality in two subjects with strokes through qualitative analysis. Nonlinear Dynamics, Psychology, and Life Sciences. 2017; 21(4):555-567. PMID: 28923161

[11] Parham LD, Mailloux Z. Sensory integration. In: CaseSmith J, O'Brien J, editors. Occupational Therapy for Children. Berlin: Elsevier; 2005.

[12] Piven E, Derakhshanrad SA. A case study demonstrating reduction of aggressive client behaviors using the Neuro-Occupation model: Addressing professional burnout through nonlinear thinking. Occupational Therapy in Mental Health. 2017; 33(2):179-94. doi: 10.1080/0164212X.2017.1278734

[13] Loukas KM. Occupational placemaking: Facilitating selforganization through use of a sensory room. Mental Health Special Interest Section Quarterly. 2011; 34(2):1-4

[14] Williams KL. Understanding the role of sensory processing in occupation: An updated discourse with cognitive neuroscience. Journal of Occupational Science. 2017; 24(3):302-13. doi: 10.1080/14427591.2016.1209425

[15] Carter RE, Lubinsky J, Domholdt E. Rehabilitation research: Principles and applications. Berlin: Elsevier; 2011

[16] Morgan. DL, Morgan. RK. Single-case research methods for the behavioral and health sciences. Thousand Oaks, California: SAGE; 2009.

[17] Creswell JW. Research design: Qualitative, quantitative, and mixed methods approaches. Thousand Oaks, California: SAGE; 2013.

[18] Eliasson AC, Krumlinde-Sundholm L, Rösblad B, Beckung E, Arner M, Öhrvall AM, et al. The Manual Ability Classification System (MACS) for children with cerebral palsy: Scale development and evidence of validity and reliability. Developmental Medicine and Child Neurology. 2006; 48(7):549-54 doi: 10.1017/S0012162206001162

[19] Bohannon RW, Smith MB. Interrater reliability of a Modified Ashworth Scale of muscle spasticity. Physical Therapy. 1987; 67(2):206-7. PMID: 3809245

[20] Park EJ, Baek SH, Park S. Systematic review of the effects of mirror therapy in children with cerebral palsy. Journal of Physical Therapy Science. 2016; 28(11):3227-31. doi: 10.1589/ jpts.28.3227

[21] Gygax MJ, Schneider P, Newman CJ. Mirror therapy in children with hemiplegia: A pilot study. Developmental Medicine \& Child Neurology. 2011; 53(5):473-6. doi: 10.1111/j.1469-8749.2011.03924.x 
[22] Grunt S, Newman CJ, Saxer S, Steinlin M, Weisstanner C, Kaelin-Lang A. The mirror illusion increases motor cortex excitability in children with and without hemiparesis. Neurorehabilitation and Neural Repair. 2017; 31(3):280-9. doi: $10.1177 / 1545968316680483$

[23] Smorenburg AR, Ledebt A, Deconinck FJ, Savelsbergh GJ. Practicing a matching movement with a mirror in individuals with spastic hemiplegia. Research in Developmental Disabilities. 2013; 34(9):2507-13. doi: 10.1016/j.ridd.2013.05.001

[24] Bruchez R, Jequier Gygax M, Roches S, Fluss J, Jacquier $\mathrm{D}$, Ballabeni $\mathrm{P}$, et al. Mirror therapy in children with hemiparesis: a randomized observer-blinded trial. Developmental Medicine \& Child Neurology. 2016; 58(9):970-8. doi: 10.1111/ dmcn.13117 
